\title{
Clustering conditions and the cluster formation process in a dynamical model of multi-dimensional attracting agents
}

\author{
F. De Smet* and D. Aeyels ${ }^{\dagger}$ \\ SYSTeMS Research Group, \\ Dept. of Electrical Energy, \\ Systems and Automation, Ghent University, \\ Technologiepark Zwijnaarde 914, \\ 9052 Zwijnaarde, Belgium
}

\begin{abstract}
We consider a multi-agent clustering model where each agent belongs to a multi-dimensional space. We investigate its long term behaviour and we prove emergence of clustering behaviour in the sense that the velocities of the agents approach asymptotic values, independently of the initial conditions; agents with equal asymptotic velocities are said to belong to the same cluster. We propose a set of relations governing these asymptotic velocities. These results are compared with results obtained earlier for the model with agents belonging to a one-dimensional space, and are then explored for the case of an infinite number of agents. For the particular case of a spherically symmetric configuration of an infinite number of agents a rigorous analysis of the relations governing the asymptotic velocities is possible, assuming that a continuity property established for the finite case remains true for the infinite case. This leads to a characterization of the onset of cluster formation in terms of the evolution of the cluster size with varying coupling strength. A remarkable point is that the cluster formation process depends critically on the dimension of the agent state space; considering the cluster size as an order parameter, the cluster formation in the one-dimensional case may be seen as a second-order phase transition, while the multi-dimensional case is associated with a first-order phase transition. We provide bounds for the critical coupling strength at the onset of the cluster formation, and we illustrate the results with two examples in three dimensions.
\end{abstract}

\footnotetext{
*fidesmet@gmail.be

†Dirk.Aeyels@UGent.be
} 


\section{INTRODUCTION}

Clustering is a phenomenon that may be observed in a variety of systems, such as animal swarms [? ? ? ? ], opinion formation processes [? ? ? ? ], and systems of coupled oscillators (e.g. pacemaker cells in the heart) [? ? ]. Cohesion of a single cluster [? ? ? ] as well as the emergence of multiple clusters have been studied [? ? ? ? ]. Depending on the specifics of the model, transitions between the different phases may be first-order [? ] or second-order [? ]. First-order phase transitions may also appear by choosing appropriate model parameters in systems that are usually characterized by a second-order phase transition [? ? ].

Most of these models are analyzed either by simulations, or by a combination of simulations and analytical results of a local nature. In [? ? ] we have introduced a model with a behavior similar to the clustering behavior of models of coupled oscillators such as the Kuramoto model [? ], but with an increased potential for analytical results concerning the long term behaviour of the solutions. The model consists of a system of mutually attracting, non-identical agents evolving in a one-dimensional state space. Each agent has a natural velocity, and is attracted to other agents by saturating interactions, i.e. the interaction between two agents converges to a constant value with increasing distance. The long term behavior depends on the coupling strength. When the coupling strength equals zero, all agents move at their natural velocities. For small values of the coupling strength several clusters arise, each characterized by the same asymptotic velocity for its members. For high values, distances between agents remain bounded, and all agents are contained in a single cluster. Our main results [? ? ? ? ] include a complete characterization of the clustering structure as it emerges with increasing time, and a complete description of the clustering formation process as a function of the coupling strength.

The model that we have introduced may be relevant for applications not related to coupled oscillators, as we have argued in [? ] for compartmental systems and in [? ] for swarming and opinion formation. The model, of which a multi-dimensional version is featured in the present paper has some distinguishing characteristics such as the range of interaction which is of a nonlocal nature, while the network representing the interaction is of a general nature. This and other aspects are detailed and discussed in [? ] in a biological context of swarming or herding.

When relating the model to swarming or opinion formation, one wonders to what extent 
the behavior of the model is limited by the restriction that agents are only able to move in one dimension. Swarms of animals obviously may have two or three dimensions at their disposal. Also the topic of opinion formation is rarely one-dimensional; it involves different interrelated aspects: e.g. conceding on one point may result in an uncompromising disposition with respect to other points.

In the present paper we focus on the multi-dimensional case: the agents belong to a Euclidean space of arbitrary dimension. As will become clear in the following sections, extensions to the multi-dimensional case of the results obtained for the one-dimensional case are not readily available -if at all possible. However, we are able to obtain a set of relations governing the asymptotic velocities of the agents; this allows us to introduce a cluster as a set of agents with the same asymptotic velocity. These relations are then explored for the case of an infinite number of agents. For the particular case of a spherically symmetric configuration of an infinite number of agents, a rigorous analysis of the relations governing the asymptotic velocities is possible, assuming that a continuity property established for the finite case remains true for the infinite case.

For more information and alternative models, see [? ] which also discusses the transformation of individual-based models into continuum models for the density of organisms, a topic we embarked on in [? ] for the case of one-dimensional agents, and which is further explored in section ??. See also [? ] for a discussion of macroscopic mathematical models for collective motion derived from a microscopic description. Our model, for the case of an infinite number of agents may be viewed as a Lagrangian representation of a continuous model; such models have been studied elsewhere, see e.g. [? ] and for a case of nonlocal interactions, see [? ]. Other rigorous results for the multi-dimensional case exist in relation to the Cucker-Smale model ([? ]) and for an infinite version, see [? ].

In the next section we introduce the multi-dimensional model, and in section ?? we review some results from [? ? ] concerning the one-dimensional model. Section ?? deals with the general model; we show that the velocities of the agents approach constant values, independently of the initial conditions. These asymptotic velocities define the different clusters. In section ?? we show that for an infinite number of agents with a spherically symmetric distribution of the natural velocities in at least two dimensions, the onset of cluster formation is akin to a first-order phase transition, as opposed to the second-order phase transition appearing in the one-dimensional case. We derive a lower and an upper bound for 
the critical value of the coupling strength, and we consider some examples corresponding to the three-dimensional case.

\section{THE MODEL}

The general model is described by

$$
\dot{x}_{i}(t)=b_{i}+K \sum_{j=1}^{N} \gamma_{j} \nabla V_{i j}\left(x_{j}(t)-x_{i}(t)\right), \quad \text { for all } i \text { in }\{1, \ldots, N\},
$$

where $K \geq 0, \gamma_{j}>0, N>1, x_{i}(t), b_{i} \in \mathbb{R}^{P}$, and $\nabla V_{i j}$ is the gradient of $V_{i j}$, with $V_{i j}$ : $\mathbb{R}^{P} \rightarrow \mathbb{R}$ assumed to be convex and twice differentiable, and $V_{j i}(y)=V_{i j}(-y)$ for all $y$ in $\mathbb{R}^{P}(i, j \in\{1, \ldots, N\})$. (Notice that this implies that $\nabla V_{i i}(0)=0$ for all $i$ in $\{1, \ldots, N\}$.)

As a special case we will consider the choice

$$
V_{i j}(y)=\int_{0}^{\|y\|} f_{i j}(\xi) \mathrm{d} \xi
$$

for all $y$ in $\mathbb{R}^{P}$, for all $i, j$ in $\{1, \ldots, N\}$, where the differentiable functions $f_{i j}: \mathbb{R}^{+} \rightarrow \mathbb{R}^{+}$ are non-decreasing with $f_{i j}(0)=0$, and satisfy

$$
f_{i j}=f_{j i}, \quad \text { and } \quad \lim _{\xi \rightarrow+\infty} f_{i j}(\xi)=F_{i j}
$$

for all $i$ and $j$ in $\{1, \ldots, N\}$, for some symmetric matrix $F \in \mathbb{R}^{N \times N}$. We will say that $f_{i j}$ saturates at the value $F_{i j}$. The resulting system is described by

$$
\dot{x}_{i}(t)=b_{i}+K \sum_{j=1}^{N} \gamma_{j} f_{i j}\left(\left\|x_{j}(t)-x_{i}(t)\right\|\right) e_{x_{j}(t)-x_{i}(t)}, \quad \text { for all } i \text { in }\{1, \ldots, N\},
$$

with

$$
e_{y} \triangleq \frac{y}{\|y\|}, \quad \text { for all } y \text { in } \mathbb{R}^{P} \backslash\{0\} ; \quad e_{0} \triangleq 0
$$

\section{PRELIMINARY RESULTS}

In this section we review results related to the model (??) for the case $P=1$ : each agent belongs to a one-dimensional space $\left(x_{i}(t) \in \mathbb{R}\right.$, for all $i$ in $\left.\{1, \ldots, N\}\right)$. For details, see [? ? ]. 


\section{A. Some notation}

For $n \in \mathbb{N}$ denote by $I_{n}$ the set $\{1, \ldots, n\}\left(I_{0} \triangleq \emptyset\right)$ and let $G=\left(G_{1}, \ldots, G_{M}\right)$ be an ordered set partition of $I_{N}$. Each set $G_{k}$ gathers the indices of the agents belonging to one cluster in a sense to be described soon. Let $G_{k}^{<}$be a shorthand notation for $\bigcup_{k^{\prime}<k} G_{k^{\prime}}$, and similarly set $G_{k}^{>} \triangleq \bigcup_{k^{\prime}>k} G_{k^{\prime}}$.

Set

$$
\widetilde{v}\left(G_{-}, G_{0}, G_{+}\right) \triangleq \frac{1}{\sum_{i \in G_{0}} \gamma_{i}} \sum_{i \in G_{0}} \gamma_{i}\left(b_{i}+K \sum_{j \in G_{+}} \gamma_{j} F_{i j}-K \sum_{j \in G_{-}} \gamma_{j} F_{i j}\right),
$$

for all $G_{-}, G_{0}, G_{+} \subset I_{N}$ with $G_{0}$ non-empty. Notice that this value corresponds to the weighted average velocity over a group of agents $G_{0}$, interacting with groups $G_{-}$and $G_{+}$, and with the interactions replaced by their saturation values.

We consider the following definition of clustering behavior of a solution $x$ of (??) with $P=1$ with respect to an ordered set partition $G$ :

- The distances between agents in the same cluster remain bounded (i.e. $\left|x_{i}(t)-x_{j}(t)\right|$ is bounded for all $i, j \in G_{k}$, for any $k \in I_{M}$, for $t \geq 0$ ).

- For any $D>0$ there exists a time after which the distances between agents in different clusters are and remain at least $D$.

- The agents are ordered by their membership to a cluster: $k<l \Rightarrow x_{i}(t)<x_{j}(t)$, $\forall i \in G_{k}, \forall j \in G_{l}, \forall t \geq T$, for some $T>0$.

Remark 1. For convenience, the definition of clustering behavior includes the order of the agents. For $P>1$ it is not clear how to order clusters or agents in an appropriate way, and later on we will consider unordered set partitions for the cluster structure of solutions of the model (??) in arbitrary dimensions.

\section{B. Results}

The following theorems are drawn from [? ]. (In Theorem ?? the matrix $F$ is restricted to be symmetric since this is required by the model (??); this restriction was not required for the model investigated in [? ].) As for Theorem ?? we also point to some peculiarities when thinking about an extension to the multi-dimensional agent case. 
Theorem 1. Consider the following inequalities

$$
\begin{aligned}
& \widetilde{v}\left(G_{k}^{<}, G_{k}, G_{k}^{>}\right)<\widetilde{v}\left(G_{k+1}^{<}, G_{k+1}, G_{k+1}^{>}\right), \quad \text { for all } k \text { in } I_{M-1}, \\
& \widetilde{v}\left(G_{k}^{<} \cup G_{k, 1}, G_{k, 2}, G_{k}^{>}\right)<\widetilde{v}\left(G_{k}^{<}, G_{k, 1}, G_{k}^{>} \cup G_{k, 2}\right), \\
& \quad \text { for all partitions }\left\{G_{k, 1}, G_{k, 2}\right\} \text { of } G_{k}, \\
& \quad \text { for all } k \text { in } I_{M} .
\end{aligned}
$$

Denote by (??'), resp. (??'), the inequalities (??), resp. (??), in which the strict inequalities are replaced by non-strict inequalities. Denote by (??) the inequalities (??) together with (??), and by (??') the inequalities (??') together with (??'). Then the conditions (??), resp. (??'), are sufficient, resp. necessary, for clustering behavior with respect to $G$ of all solutions of the system (??) with $P=1$.

The conditions (??) and (??') are related to the property that the distances between different clusters grow unbounded, while (??) and (??') express that for any partition of a cluster into two subsets, the two components cannot separate and therefore the distances between agents belonging to this cluster remain bounded. Notice that separate conditions are not sufficient to draw conclusions about the behavior of one or two individual clusters: the complete set of conditions (??) should be considered.

Theorem 2. For every $b \in \mathbb{R}^{N}$ and every symmetric matrix $F \in\left(\mathbb{R}^{+}\right)^{N \times N}$, there exists a partition of $\mathbb{R}^{+}$in a finite number of intervals, such that each interval corresponds to a unique ordered set partition $G$ of $I_{N}$, for which (??) holds for all $K$ in the interior of this interval.

When the coupling strength $K$ varies and one of the corresponding inequalities (??) is no longer satisfied, the cluster structure will change. Depending on whether one of the inequalities (??) or (??) is violated, either two clusters will merge and form a new cluster, or one cluster will split into two clusters. In case multiple inequalities turn simultaneously into an equality for a particular value of $K$, multiple clusters will be affected, but this transition is not robust: in general, a perturbation in the other model parameters $\left(b_{i}, \gamma_{i}\right.$, or $\left.F_{i j}\right)$ will split up the transition in several 'elementary' transitions, taking place at different values for $K$; for each elementary transition a cluster either splits into two new clusters or a new cluster is formed by merging two clusters. Compare this to the case $P>1$ where there may be robust transitions involving more than two clusters as was illustrated in [? ]. In this 
case small perturbations in the model parameters $b_{i}, \gamma_{i}$, or $F_{i j}$ only result in a shift for the transition value for $K$ and for the resulting asymptotic velocities; the same clusters will be involved in the transition. This is illustrated in Fig. ??, where for specific examples — with all-to-all coupling, i.e. $f_{i j}$ is independent of $i$ and $j$, equal weights $\gamma_{i}$, and the parameters $b_{i}$ chosen arbitrarily - the evolution of the asymptotic velocities $v_{i}$ of the agents (horizontal axis/plane) are shown (see also Theorems ?? and ??, stating that the velocities of the agents indeed converge to asymptotic values) with varying coupling strength $K$ (vertical axis) for both $P=1$ and $P=2$. For $P=1$, each transition with increasing $K$ corresponds to merging two clusters, while for $P=2$ there is a transition corresponding to merging 6 clusters, even though the parameter values $b_{i}$ were chosen arbitrarily.

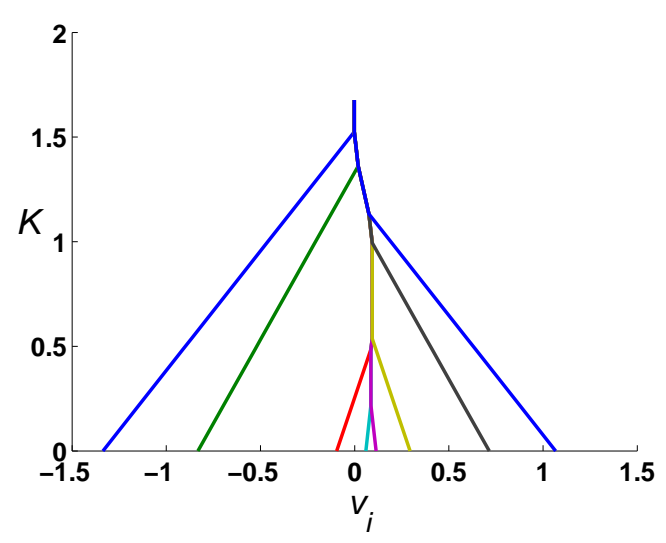

(a) $P=1$

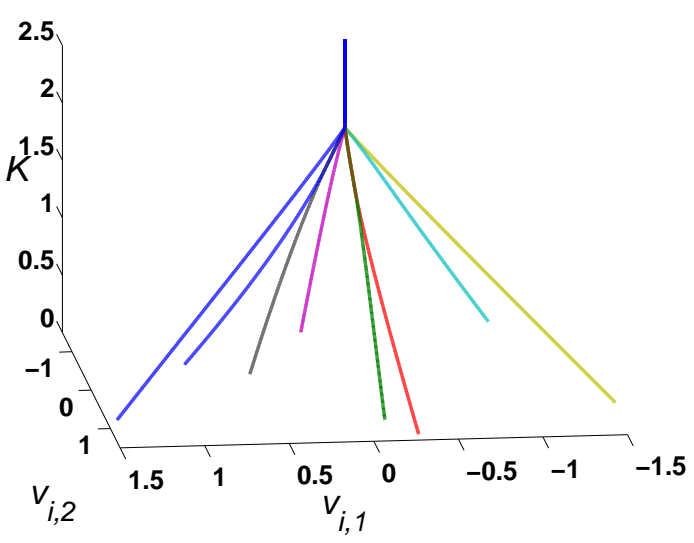

(b) $P=2$

Figure 1. Comparison of the one-dimensional case ?? with the two-dimensional case ?? for the evolution of the asymptotic velocities $v_{i}$ of the agents (horizontal axis/plane) with varying coupling strength $K$ (vertical axis). Different colors are for emphasis: they correspond to different clusters of agents. In the one-dimensional case each transition only affects 2 clusters, whereas the twodimensional case exhibits a transition involving 6 clusters.

Theorem 3. Let $x$ be a solution of (??) with $P=1$ and with cluster structure $G=$ $\left(G_{1}, \ldots, G_{M}\right)$, and assume that all functions $f_{i j}$ are increasing in $\mathbb{R}^{+}$. For each $k \in I_{M}$, if $i, j \in G_{k}$, then

$$
\begin{aligned}
& \lim _{t \rightarrow+\infty}\left(x_{i}(t)-x_{j}(t)\right) \text { exists and is independent of } x(0), \\
& \lim _{t \rightarrow+\infty} \dot{x}_{i}(t) \text { exists and equals } \widetilde{v}\left(G_{k}^{<}, G_{k}, G_{k}^{>}\right) .
\end{aligned}
$$


In other words, within a cluster all differences between $x_{i}$-values will approach constants, which are independent of the initial condition, and the velocities of the agents will approach the asymptotic average cluster velocity.

\section{MULTI-DIMENSIONAL AGENTS}

\section{A. Clustering behavior}

As mentioned before, the notion of clustering behavior defined in the previous section cannot simply be extended to the multi-dimensional case: the ordering of the clusters will be dropped in the definition. Furthermore, we will distinguish between distance clustering behavior and velocity clustering behavior.

\section{Distance clustering behavior}

A solution $x$ of (??) exhibits distance clustering behavior with respect to the (unordered) set partition $G$ if the following conditions are satisfied (we retain the defining conditions of the case $P=1$, but omit the ordering condition):

- The distances between agents in the same cluster remain bounded (i.e. $\left\|x_{i}(t)-x_{j}(t)\right\|$ is bounded for all $i, j \in G_{k}$, for any $G_{k} \in G$, for $t \geq 0$ ).

- For any $D>0$ there exists a time after which the distances between agents in different clusters are and remain at least $D$.

We have not succeeded in proving a theorem analogous to Theorem ??, mainly because the expressions defining the cluster velocities for a given cluster structure (see e.g. Eq. (??) later on) are much harder to deal with for the case $P>1$. However, we have derived general analytical results about the asymptotic behavior of solutions of the system (??) for $P>1$, which relate to the notion of asymptotic velocity clustering behavior, to be introduced next. Our results for $P>1$, when compared to the second result of Theorem ??, lack an explicit formula for the asymptotic velocities but do not require the a priori assumption of clustering behavior. The proof of the first result of Theorem (??) is easily extended to the case $P>1$ if one assumes that the solution $x$ exhibits distance clustering behavior. However, since we 
have no proof ensuring distance clustering behavior, we will not elaborate on this and we will mainly focus on asymptotic velocity clustering.

\section{Velocity clustering behavior}

A solution $x$ of (??) is said to exhibit asymptotic velocity clustering behavior with respect to a set partition $H$ of $I_{N}$ if $\lim _{t \rightarrow+\infty} \dot{x}_{i}(t)$ exists for all $i$ in $I_{N}$, and all agents in the same set in $H$ have the same limit value, while agents from different sets have different limit values.

We will show below (see Theorem ??) that each velocity $\dot{x}_{i}(t)$ converges for $t \rightarrow+\infty$, and as a result any solution $x$ of (??) exhibits asymptotic velocity clustering behavior with respect to some partition $H$. The results formulated in the previous section for the case $P=1$ indicate that the solutions of the system (??) (and probably also those of (??)) almost always exhibit distance clustering behavior with respect to the same cluster structure as the asymptotic velocity cluster structure, and only for some non-generic parameter values may these cluster structures be different (or may there be no distance clustering behavior).

Even though we do not prove the occurrence of distance clustering behavior, it is easily verified that if a solution $x$ of (??) exhibits distance clustering behavior with respect to a partition $G$, then it follows that $G$ is a refinement of the asymptotic velocity cluster structure $H$, i.e. $\forall G_{k} \in G: \exists H_{l} \in H, G_{k} \subset H_{l}$, since a bounded distance between two agents implies that they cannot have different asymptotic velocities. As mentioned before, in general $G$ will be equal to $H$.

Remark 2. Some systems, such as the Kuramoto model [? ? ], are believed to also exhibit distance clustering behavior, but without convergence of the velocities for $t \rightarrow+\infty$. A more general notion of average velocity clustering behavior, characterized by the limit values $\lim _{t \rightarrow+\infty} \frac{x_{i}(t)}{t}$ may therefore be introduced to include these systems. One can verify

that if $\lim _{t \rightarrow+\infty} \dot{x}_{i}(t)$ exists, then $\lim _{t \rightarrow+\infty} \frac{x_{i}(t)}{t}$ also exists and both limit values are equal, and therefore asymptotic velocity clustering behavior implies average velocity clustering behavior. For the remainder of this paper we will only consider asymptotic velocity clustering behavior (in addition to distance clustering behavior), which we will refer to as 'velocity clustering behavior'. 


\section{B. Asymptotic velocities}

We will show that the velocities of the agents converge to limit values, obtained as the solution of a minimization problem. Write (??) as

$$
\dot{x}(t)=\mathcal{F}(x(t)),
$$

for all $t$ in $\mathbb{R}$, with

$$
\mathcal{F}:\left(\mathbb{R}^{P}\right)^{N} \rightarrow\left(\mathbb{R}^{P}\right)^{N}: x \mapsto \mathcal{F}(x)=\left(\mathcal{F}_{1}(x), \ldots, \mathcal{F}_{N}(x)\right),
$$

defined by

$$
\mathcal{F}_{i}(x) \triangleq b_{i}+K \sum_{j \in I_{N}} \gamma_{j} \nabla V_{i j}\left(x_{j}-x_{i}\right)
$$

for all $x$ in $\left(\mathbb{R}^{P}\right)^{N}$, and for any $v \in\left(\mathbb{R}^{P}\right)^{N}$ consider the function

$$
\mathcal{V}_{v}:\left(\mathbb{R}^{P}\right)^{N} \rightarrow \mathbb{R}: x \mapsto \mathcal{V}_{v}(x) \triangleq-\sum_{i \in I_{N}} \gamma_{i} v_{i}^{T} x_{i}+\frac{K}{2} \sum_{i, j \in I_{N}} \gamma_{i} \gamma_{j} V_{i j}\left(x_{j}-x_{i}\right) .
$$

The derivative of $\mathcal{V}_{v}$ with respect to $x_{i} \in \mathbb{R}^{P}$ (i.e. the gradient with respect to $x_{i}$ ) can be calculated as

$$
\begin{aligned}
\frac{\partial \mathcal{V}_{v}}{\partial x_{i}}(x) & =-\gamma_{i} v_{i}-K \sum_{j \in I_{N}} \gamma_{i} \gamma_{j} \nabla V_{i j}\left(x_{j}-x_{i}\right) \\
& =-\gamma_{i}\left(v_{i}-b_{i}+\mathcal{F}_{i}(x)\right)
\end{aligned}
$$

for all $x$ in $\left(\mathbb{R}^{P}\right)^{N}$.

Remark 3 . When we pick $v=b$, then for a solution $x$ of (??), $\frac{\partial \mathcal{V}_{b}}{\partial x_{i}}(x(t))=-\gamma_{i} \dot{x}_{i}(t)$, for all $t$ in $\mathbb{R}$, for all $i$ in $I_{N}$, and as a result, $\mathcal{V}_{b}$ is a gradient function for the system (??).

The time-derivative of $\mathcal{V}_{v}$ along a solution $x$ of (??) satisfies

$$
\frac{\mathrm{d}\left(\mathcal{V}_{v} \circ x\right)}{\mathrm{d} t}(t)=-\sum_{i \in I_{N}} \gamma_{i}\left(v_{i}-b_{i}+\mathcal{F}_{i}(x(t))\right)^{T} \mathcal{F}_{i}(x(t))
$$

which may be rewritten as

$$
=\frac{1}{2} \sum_{i \in I_{N}} \gamma_{i}\left(\left\|v_{i}-b_{i}\right\|^{2}-\left\|\mathcal{F}_{i}(x(t))\right\|^{2}-\left\|v_{i}-b_{i}+\mathcal{F}_{i}(x(t))\right\|^{2}\right),
$$


for all $t$ in $\mathbb{R}$. Introducing the function

$$
\mathcal{Q}:\left(\mathbb{R}^{P}\right)^{N} \rightarrow \mathbb{R}: x \mapsto \mathcal{Q}(x) \triangleq \sum_{i \in I_{N}} \gamma_{i}\left\|x_{i}\right\|^{2}
$$

which defines the square of a norm of a vector in $\left(R^{P}\right)^{N}$ :

$$
\forall x \in\left(R^{P}\right)^{N}:\|x\| \triangleq \sqrt{\mathcal{Q}(x)}
$$

we may write

$$
\begin{aligned}
\frac{\mathrm{d}\left(\mathcal{V}_{v} \circ x\right)}{\mathrm{d} t}(t) & =\frac{1}{2}\left(\|v-b\|^{2}-\|\mathcal{F}(x(t))\|^{2}-\|v-b+\mathcal{F}(x(t))\|^{2}\right) \\
& =\frac{1}{2}(\mathcal{Q}(v-b)-\mathcal{Q}(\mathcal{F}(x(t)))-\mathcal{Q}(v-b+\mathcal{F}(x(t)))) .
\end{aligned}
$$

We may therefore pick any $v$ such that $\mathcal{Q}(v-b) \leq \inf _{x \in\left(\mathbb{R}^{P}\right)^{N}} \mathcal{Q}(\mathcal{F}(x))$ and obtain a function $\mathcal{V}_{v}$ that does not increase along solutions of (??). However, since solutions of (??) may not be bounded, a direct application of Lyapunov theory is not possible.

Moreover, if $\mathcal{Q}(v-b)<\inf _{x \in\left(\mathbb{R}^{P}\right)^{N}} \mathcal{Q}(\mathcal{F}(x))$, the derivative $\frac{\mathrm{d}\left(\mathcal{V}_{v} \circ x\right)}{\mathrm{d} t}(t)$ will not converge to zero. In order to obtain a function $\mathcal{V}_{v}$ that is non-increasing along solutions of (??) while its time-derivative may converge to zero, we need to consider values $v \in\left(\mathbb{R}^{P}\right)^{N}$ for which $\mathcal{Q}(v-b)=\inf _{x \in\left(\mathbb{R}^{P}\right)^{N}} \mathcal{Q}(\mathcal{F}(x))$.

More specifically we will consider the function $\mathcal{V}_{b-\bar{v}}$ where $\bar{v}$ is defined as the value for $v$ in $\overline{\operatorname{im}(\mathcal{F})}$ (i.e. $v$ belongs to the closure in $\left(\mathbb{R}^{P}\right)^{N}$ of the image of $\left.\mathcal{F}\right)$ minimizing $\mathcal{Q}(v)$. Because $\mathcal{F}$ may be considered as the gradient of the convex function $\mathcal{V}_{b}$, the interior of $\operatorname{im}(\mathcal{F})=\mathcal{F}\left(\left(\mathbb{R}^{P}\right)^{N}\right)$ (and therefore also its closure) is a convex set (see [?]). Since the function $\mathcal{Q}$ is strictly convex and radially unbounded, it follows that $\bar{v}$ is unique as the minimizer of $\mathcal{Q}$ over the set $\overline{\operatorname{im}(\mathcal{F})}$.

Remark 4 . Notice that since both $\mathcal{Q}$ and $\overline{\operatorname{im}(\mathcal{F})}$ vary continuously with the model parameters, $\bar{v}$ is also continuous in the model parameters.

Although $\mathcal{V}_{b-\bar{v}}(x)$ may not be bounded from below, we will show in Theorem ?? that for large $\|x\|$, it decreases slower than linearly in $\|x\|$; this together with (??) will allow us to conclude that $\lim _{t \rightarrow+\infty} \dot{x}(t)=\bar{v}$.

First we show that also $\mathcal{Q}$ is non-increasing along solutions of (??). We denote by $\Psi_{i j}$ the Hessian matrix of $V_{i j}:\left(\Psi_{i j}\right)_{m n}=\frac{\partial^{2} V_{i j}}{\partial y_{m} \partial y_{n}}$, for all $i, j$ in $I_{N}$ and $m, n$ in $I_{P}$. Then the 
time-derivative of $\mathcal{Q} \circ \mathcal{F}$ along a solution $x$ of (??) satisfies

$$
\begin{aligned}
\frac{\mathrm{d}(\mathcal{Q} \circ \mathcal{F} \circ x)}{\mathrm{d} t}(t) & =2 \sum_{i, j \in I_{N}} \gamma_{i} \gamma_{j} \mathcal{F}_{i}(x(t))^{T} K \Psi_{i j}\left(x_{j}(t)-x_{i}(t)\right)\left(\dot{x}_{j}(t)-\dot{x}_{i}(t)\right) \\
& =-K \sum_{i, j \in I_{N}} \gamma_{i} \gamma_{j}\left(\dot{x}_{j}(t)-\dot{x}_{i}(t)\right)^{T} \Psi_{i j}\left(x_{j}(t)-x_{i}(t)\right)\left(\dot{x}_{j}(t)-\dot{x}_{i}(t)\right) \\
& \leq 0
\end{aligned}
$$

for all $t$ in $\mathbb{R}$, since the matrices $\Psi_{i j}(y)$ are all positive semi-definite because of the convexity of the functions $V_{i j}$. (Although $\mathcal{Q} \circ \mathcal{F}$ is bounded from below since it is non-negative, and may therefore be considered as a Lyapunov function, Lyapunov theory does not apply directly since a solution $x$ may — and generally will — be unbounded.)

As a result, for any solution $x$ of $(? ?),\|\dot{x}(t)\|$ is non-increasing in $t$, and as we will show in Theorem ?? it converges to $\|\bar{v}\|=\min _{v \in \overline{\operatorname{im}(\mathcal{F})}}\|v\|$ as $t \rightarrow+\infty$. The proof will be based on the non-increasing behavior of $\mathcal{V}_{b-\bar{v}}$ and $\mathcal{Q} \circ \mathcal{F}$ along solutions of (??), and on the behavior of $\mathcal{V}_{b-\bar{v}}(x)$ for large values of $\|x\|$.

Theorem 4. Any solution $x$ of (??) satisfies

$$
\lim _{t \rightarrow+\infty} \dot{x}(t)=\bar{v}
$$

Proof. By (??) the time-derivative of the function $\mathcal{V}_{b-\bar{v}}$ along a solution $x$ of (??) equals

$$
\begin{aligned}
\frac{\mathrm{d}\left(\mathcal{V}_{b-\bar{v}} \circ x\right)}{\mathrm{d} t}(t) & =\frac{1}{2}(\mathcal{Q}(\bar{v})-\mathcal{Q}(\dot{x}(t))-\mathcal{Q}(\bar{v}-\dot{x}(t))) \\
& \leq 0
\end{aligned}
$$

for all $t$ in $\mathbb{R}$, since $\mathcal{Q}(\bar{v}) \leq \mathcal{Q}(\dot{x}(t))$ because of the definition of $\bar{v}$.

As noticed before, Lyapunov theory does not apply, but we will still be able to show that

$$
\limsup _{t \rightarrow+\infty} \frac{\mathrm{d}\left(\mathcal{V}_{b-\bar{v}} \circ x\right)}{\mathrm{d} t}(t)=0
$$

It then follows that

$$
\limsup _{t \rightarrow+\infty}(\mathcal{Q}(\bar{v})-\mathcal{Q}(\dot{x}(t)))=0 .
$$

Since $\mathcal{Q}(\dot{x}(t))$ is non-increasing in $t, \lim _{t \rightarrow+\infty} \mathcal{Q}(\dot{x}(t))=\mathcal{Q}(\bar{v})$, from which we conclude that $\lim _{t \rightarrow+\infty} \dot{x}(t)=\bar{v}$, since otherwise there would exist a (positive) limit point $\bar{w}$ of $\dot{x}$ different from $\bar{v}$ (since $\|\dot{x}(t)\|$ is non-increasing in $t$, it remains bounded) with $\mathcal{Q}(\bar{w})=\mathcal{Q}(\bar{v})$, contradicting the uniqueness of $\bar{v}$ as the minimizer of $\mathcal{Q}$ over $\overline{\operatorname{im}(\mathcal{F})}$. 
We will now prove (??). Assume that

$$
\limsup _{t \rightarrow+\infty} \frac{\mathrm{d}\left(\mathcal{V}_{b-\bar{v}} \circ x\right)}{\mathrm{d} t}(t)=-\epsilon, \quad \text { for some } \epsilon>0 .
$$

Then

$$
\limsup _{t \rightarrow+\infty} \frac{\mathcal{V}_{b-\bar{v}}(x(t))}{t} \leq-\epsilon
$$

and thus $\lim _{t \rightarrow+\infty} \mathcal{V}_{b-\bar{v}}(x(t))=-\infty$ implying that $\lim _{t \rightarrow+\infty}\|x(t)\|=+\infty$. Since $\mathcal{Q} \circ \mathcal{F} \circ x$ is non-increasing, $\|\dot{x}(t)\|$ is bounded for $t \geq 0$ by $C \triangleq\|\dot{x}(0)\|$, and $\lim \sup _{t \rightarrow+\infty} \frac{\|x(t)\|}{t} \leq C$. It follows that

$$
\limsup _{t \rightarrow+\infty} \frac{\mathcal{V}_{b-\bar{v}}(x(t))}{\|x(t)\|} \leq-\frac{\epsilon}{C}
$$

Consider $\bar{x}^{n} \in\left(\mathbb{R}^{P}\right)^{N}(n \in \mathbb{N})$ with $\lim _{n \rightarrow \infty} \mathcal{F}\left(\bar{x}^{n}\right)=\bar{v}$. Because of the convexity of $\mathcal{V}_{b-\bar{v}}$, the graph of $\mathcal{V}_{b-\bar{v}}$ is bounded from below by the tangent hyperplane in $\bar{x}^{n}$ :

$$
\begin{aligned}
\mathcal{V}_{b-\bar{v}}(x) & \geq \mathcal{V}_{b-\bar{v}}\left(\bar{x}^{n}\right)+\sum_{i \in I_{N}} \gamma_{i}\left(x_{i}-\bar{x}_{i}^{n}\right)^{T}\left(\bar{v}_{i}-\mathcal{F}_{i}\left(\bar{x}^{n}\right)\right) \\
& \geq \mathcal{V}_{b-\bar{v}}\left(\bar{x}^{n}\right)-\left\|x-\bar{x}^{n}\right\|\left\|\bar{v}-\mathcal{F}\left(\bar{x}^{n}\right)\right\|
\end{aligned}
$$

for all $x$ in $\left(\mathbb{R}^{P}\right)^{N}$. Consequently, since a solution $x$ of (??) satisfies $\lim _{t \rightarrow+\infty}\|x(t)\|=+\infty$,

$$
\limsup _{t \rightarrow+\infty} \frac{\mathcal{V}_{b-\bar{v}}(x(t))}{\|x(t)\|} \geq-\left\|\bar{v}-\mathcal{F}\left(\bar{x}^{n}\right)\right\|
$$

and for $n$ sufficiently large we obtain a contradiction with (??).

We conclude that $\lim \sup _{t \rightarrow+\infty} \frac{\mathrm{d}\left(\mathcal{V}_{b-\bar{v}} \circ x\right)}{\mathrm{d} t}(t)=0$, and that $\lim _{t \rightarrow+\infty} \dot{x}_{i}(t)=\bar{v}_{i}$, for all $i$ in $I_{N}$

Considering the limit $t \rightarrow+\infty$ in the special case (??), and taking into account that

$$
\lim _{t \rightarrow+\infty} e_{x_{j}(t)-x_{i}(t)}=\lim _{t \rightarrow+\infty} \frac{\frac{x_{j}(t)}{t}-\frac{x_{i}(t)}{t}}{\left\|\frac{x_{j}(t)}{t}-\frac{x_{i}(t)}{t}\right\|}=e_{\bar{v}_{j}-\bar{v}_{i}}
$$

whenever $\bar{v}_{i} \neq \bar{v}_{j}\left(i, j \in I_{N}\right)$, we may calculate the weighted average of (??) over the velocity clusters $H_{k}$ to obtain that

$$
\bar{v}_{i}=\frac{1}{\sum_{l \in H_{k}} \gamma_{l}} \sum_{l \in H_{k}} \gamma_{l}\left(b_{l}+K \sum_{j \in I_{N}} \gamma_{j} F_{l j} e_{\bar{v}_{j}-\bar{v}_{l}}\right),
$$

for all $i$ in $H_{k}$, for all $k \in I_{M}$. (Recall that $e_{0}=0$ by definition.) The existence of velocities $\bar{v}_{i}$ satisfying these equations may be considered as the equivalent of (??) for $P>1$, and it 
immediately follows that (??) is a necessary condition for velocity clustering behavior with respect to $H$.

The equivalent of (??) would be the absence of a solution for $\bar{v}$ to (??) for any refinement $\tilde{H}$ of $H$ with $\tilde{H} \neq H$. Unlike the partitions in only two subsets considered in the conditions (??), any refinement of $H$ has to be taken into consideration. This is illustrated in [? ], where we showed that even for generic parameter values a cluster may split into three subsets when decreasing the coupling strength $K$, without an intermediate range for $K$ where two clusters exist. For these parameter values there will be a solution to (??) with respect to a partition into three subsets, but there may be no solutions with respect to partitions into two subsets.

\section{Independence of the initial condition}

Theorem ?? implies that the asymptotic velocities (and therefore also the velocity clusters) are independent of the initial condition. In this section we show that for solutions of (??) exhibiting distance clustering behavior, the distance cluster structure is also independent of the initial condition. This follows from similar arguments as in [? ? ]; we consider two solutions $x^{*}$ and $x$ and we show that the function $\mathcal{Q} \circ\left(x-x^{*}\right)$ is non-increasing. First notice that for any $a, b \in \mathbb{R}^{P}$ and $i, j \in I_{N}$,

$$
\begin{aligned}
& (a-b)^{T}\left(\nabla V_{i j}(a)-\nabla V_{i j}(b)\right) \\
& \quad=(a-b)^{T} \int_{0}^{1} \mathrm{~d} t \Psi_{i j}(b+t(a-b))(a-b) \\
& \quad \geq 0
\end{aligned}
$$

(because $\Psi_{i j}$ is positive semi-definite), and therefore

$$
\begin{aligned}
& \frac{\mathrm{d}\left(\mathcal{Q} \circ\left(x-x^{*}\right)\right)}{\mathrm{d} t}(t)= 2 K \sum_{i, j \in I_{N}} \gamma_{i} \gamma_{j}\left(x_{i}(t)-x_{i}^{*}(t)\right)^{T} \\
& \times\left(\nabla V_{i j}\left(x_{j}(t)-x_{i}(t)\right)-\nabla V_{i j}\left(x_{j}^{*}(t)-x_{i}^{*}(t)\right)\right) \\
&= K \sum_{i, j \in I_{N}}\left(x_{j}(t)-x_{i}(t)-x_{j}^{*}(t)+x_{i}^{*}(t)\right)^{T} \\
& \times\left(\nabla V_{i j}\left(x_{j}(t)-x_{i}(t)\right)-\nabla V_{i j}\left(x_{j}^{*}(t)-x_{i}^{*}(t)\right)\right) \\
& \leq 0 .
\end{aligned}
$$


Consequently $\mathcal{Q}\left(x(t)-x^{*}(t)\right)$ and $\left\|x_{i}(t)-x_{i}^{*}(t)\right\|\left(i \in I_{N}\right)$ remain bounded when $t \rightarrow+\infty$, and therefore distance clustering behavior of $x^{*}$ implies distance clustering behavior of $x$ with respect to the same cluster structure.

\section{Convexity of the clusters for all-to-all coupling}

In this section we restrict the interaction structure to all-to-all coupling, i.e. we assume that the functions $V_{i j}$ are all equal to a function denoted by $V_{0}$, which is convex and satisfies $V_{0}(-y)=V_{0}(y)$, for all $y$ in $\mathbb{R}^{P}$, and which we assume to be radially unbounded. (Notice that this implies that $\nabla V_{0}(0)=0$.)

We consider a solution exhibiting distance or velocity clustering behavior with respect to a given cluster structure, and for an agent with a natural velocity which is a convex combination of natural velocities of agents belonging to a common cluster, we show that this agent also belongs to this cluster. This property does not hold when there is no all-to-all coupling (see e.g. [? , section 5(b)] for an example with $P=1$ ). For distance clustering behavior we follow a similar reasoning as in [? ], where this property was shown for the system (??); for velocity clustering behavior the proof requires some modifications.

In section ?? we investigate a bound for the asymptotic velocity difference for a velocity cluster and an agent not belonging to this velocity cluster, and therefore having a natural velocity that is not in the convex hull of the natural velocities of the agents in the cluster.

\section{Convexity of the distance clusters}

Assume the solution $x$ of (??) exhibits distance clustering behavior with respect to $G=$ $\left\{G_{1}, \ldots, G_{M}\right\}$, with $b_{m}=\sum_{j \in G_{k}} \mu_{j} b_{j}$, for some $k \in I_{M}, m \in I_{N}, \mu \in[0,1]^{N}$ with $\mu_{j}=0$ if $j \notin G_{k}, \mu_{m}=0$, and $\sum_{j \in I_{N}} \mu_{j}=\sum_{j \in G_{k}} \mu_{j}=1$. We will show that this convexity property implies that $m \in G_{k}$. Consider the function $W$, defined by

$$
W: \mathbb{R} \rightarrow \mathbb{R}: t \mapsto W(t) \triangleq \frac{1}{2}\left\|x_{m}(t)-\sum_{j \in G_{k}} \mu_{j} x_{j}(t)\right\|^{2}
$$


Then

$$
\begin{aligned}
\frac{\mathrm{d} W}{\mathrm{~d} t}(t)= & \left(x_{m}(t)-\sum_{j \in G_{k}} \mu_{j} x_{j}(t)\right)^{T}\left(\mathcal{F}_{m}(x(t))-\sum_{l \in G_{k}} \mu_{l} \mathcal{F}_{l}(x(t))\right) \\
= & \sum_{l \in G_{k}} \mu_{l}\left(x_{m}(t)-x_{l}(t)\right)^{T}\left(\mathcal{F}_{m}(x(t))-\mathcal{F}_{l}(x(t))\right) \\
& \quad+\sum_{j \in G_{k}} \sum_{l \in G_{k}} \mu_{j} \mu_{l}\left(x_{l}(t)-x_{j}(t)\right)^{T}\left(\mathcal{F}_{m}(x(t))-\mathcal{F}_{l}(x(t))\right) .
\end{aligned}
$$

In the second term $\left\|\mathcal{F}_{m}(x(t))-\mathcal{F}_{l}(x(t))\right\|$ is bounded by some constant $C_{0}$ since $\|\mathcal{F}(x(t))\|=$ $\sqrt{\mathcal{Q}(\mathcal{F}(x(t)))}$ is non-increasing in $t$. In the first term we rewrite

$$
\begin{aligned}
&\left(x_{m}(t)-x_{l}(t)\right)^{T}\left(\mathcal{F}_{m}(x(t))-\mathcal{F}_{l}(x(t))\right)=\sum_{n \in I_{N}}\left(x_{m}(t)-x_{n}(t)+x_{n}(t)-x_{l}(t)\right) \\
& \times K \gamma_{n}\left(\nabla V_{0}\left(x_{n}(t)-x_{m}(t)\right)-\nabla V_{0}\left(x_{n}(t)-x_{l}(t)\right)\right),
\end{aligned}
$$

and we apply the property (??) for $n \neq m$ and $n \neq l$, resulting in

$$
\begin{aligned}
\frac{\mathrm{d} W}{\mathrm{~d} t}(t) \leq- & K \sum_{l \in G_{k}} \mu_{l}\left(\gamma_{m}+\gamma_{l}\right)\left(x_{m}(t)-x_{l}(t)\right)^{T} \nabla V_{0}\left(x_{m}(t)-x_{l}(t)\right) \\
& +C_{0} \sum_{j \in G_{k}} \sum_{l \in G_{k}} \mu_{j} \mu_{l}\left\|x_{l}(t)-x_{j}(t)\right\| .
\end{aligned}
$$

Since (using the convexity of $V_{0}$ )

$$
y^{T} \nabla V_{0}(y)=\left.\frac{\partial\left(V_{0}(t y)\right)}{\partial t}\right|_{t=1} \geq V_{0}(y)-V_{0}(0)
$$

for all $y$ in $\mathbb{R}^{P}$, we obtain that

$$
\begin{aligned}
\frac{\mathrm{d} W}{\mathrm{~d} t}(t) \leq- & K \sum_{l \in G_{k}} \mu_{l}\left(\gamma_{m}+\gamma_{l}\right) V_{0}\left(x_{m}(t)-x_{l}(t)\right)+C_{1} \\
& +C_{0} \sum_{j \in G_{k}} \sum_{l \in G_{k}} \mu_{j} \mu_{l}\left\|x_{l}(t)-x_{j}(t)\right\|
\end{aligned}
$$

where $C_{1} \triangleq K \sum_{l \in G_{k}} \mu_{l}\left(\gamma_{m}+\gamma_{l}\right) V_{0}(0)$.

Because of the distance clustering behavior $\left\|x_{l}(t)-x_{j}(t)\right\|$ (with $j, l \in G_{k}$ ) is bounded, and since $V_{0}$ is radially unbounded it follows that for some $\delta>0$ we can find a constant $D$ such that, if $W(t)>D$, then (taking into account that $G_{k}$ is a distance cluster) $\frac{\mathrm{d} W}{\mathrm{~d} t}(t)<-\delta$, implying that $W(t) \leq D$ for $t$ sufficiently large and therefore $m \in G_{k}$. 


\section{Convexity of the velocity clusters}

We denote the velocity cluster structure by $H=\left\{H_{1}, \ldots, H_{M}\right\}$, and similarly as in the previous section, we assume that $b_{m}=\sum_{j \in H_{k}} \mu_{j} b_{j}$, for some $k \in I_{M}, m \in I_{N}, \mu \in[0,1]^{N}$ with $\mu_{j}=0$ if $j \notin H_{k}, \mu_{m}=0$, and $\sum_{j \in I_{N}} \mu_{j}=\sum_{j \in H_{k}} \mu_{j}=1$. We will show that $m \in H_{k}$, using the function $W$, now defined by

$$
W: \mathbb{R} \rightarrow \mathbb{R}: t \mapsto W(t) \triangleq \frac{1}{2}\left\|x_{m}(t)-\sum_{j \in H_{k}} \mu_{j} x_{j}(t)\right\|^{2}
$$

Repeating the reasoning in the previous section, but applying the property (??) for all $n$ in (??), we obtain

$$
\frac{\mathrm{d} W}{\mathrm{~d} t}(t) \leq C_{0} \sum_{j \in H_{k}} \sum_{l \in H_{k}} \mu_{j} \mu_{l}\left\|x_{l}(t)-x_{j}(t)\right\| .
$$

Since the velocity clustering behavior implies that $\lim _{t \rightarrow+\infty} \frac{\left\|x_{l}(t)-x_{j}(t)\right\|}{t}=0$ for $j, l$ in $H_{k}$, it follows that for any $\epsilon>0$ we can find a constant $T$ such that $\left\|x_{l}(t)-x_{j}(t)\right\| \leq \epsilon t$ for all $t>T$, and for all $j, l$ in $H_{k}$, and therefore

$$
\frac{\mathrm{d} W}{\mathrm{~d} t}(t) \leq C_{0} \epsilon t, \quad \text { for all } t>T,
$$

resulting in

$$
W(t) \leq C_{2}+\frac{C_{0}}{2} \epsilon t^{2}, \quad \text { for all } t>T
$$

for some $C_{2} \in \mathbb{R}$, and

$$
\left\|\bar{v}_{m}-\sum_{j \in H_{k}} \mu_{j} \bar{v}_{j}\right\|=\lim _{t \rightarrow+\infty} \frac{\sqrt{2 W(t)}}{t} \leq \sqrt{C_{0} \epsilon} .
$$

Since this propery holds for all $\epsilon>0$, we conclude that $\bar{v}_{m}=\bar{v}_{j}$ with $j \in H_{k}$, and therefore that $m \in H_{k}$.

\section{Upper bound for asymptotic velocity differences}

We consider again a solution $x$ of (??) with all-to-all coupling, and with the velocity cluster structure of $x$ denoted by $H=\left\{H_{1}, \ldots, H_{M}\right\}$. If agent $m \in I_{N}$ does not belong to the cluster $H_{k}$, then it follows from the previous section that $b_{m}$ does not belong to the convex hull of the natural velocities of the agents belonging $H_{k}$. In order to provide a bound 
for the asymptotic velocity difference $\left\|\bar{v}_{m}-\bar{v}_{j}\right\|$ with $j \in H_{k}$, we first prove the following lemma.

Lemma 1. Let $x$ be a solution of the system (??) with $V_{i j}=V_{0}$ for all $i, j$ in $I_{N}$ for some convex function $V_{0}$. Set $\lim _{t \rightarrow+\infty} \dot{x}(t)=\bar{v}$. (See Theorem ??.) Then

$$
\left\|\bar{v}_{i}-\bar{v}_{j}\right\| \leq\left\|b_{i}-b_{j}\right\|
$$

for all $i, j$ in $I_{N}$.

Proof. Consider the function

$$
W: \mathbb{R} \rightarrow \mathbb{R}: t \mapsto W(t) \triangleq \frac{1}{2}\left\|x_{i}(t)-x_{j}(t)\right\|^{2} .
$$

Then

$$
\begin{aligned}
\frac{\mathrm{d} W}{\mathrm{~d} t}(t)=\left(x_{i}(t)-x_{j}(t)\right)^{T}\left(b_{i}-b_{j}\right. \\
\left.\quad+K \sum_{n \in I_{N}} \gamma_{n}\left(\nabla V_{0}\left(x_{n}(t)-x_{i}(t)\right)-\nabla V_{0}\left(x_{n}(t)-x_{j}(t)\right)\right)\right) \\
=\left(x_{i}(t)-x_{j}(t)\right)^{T}\left(b_{i}-b_{j}\right) \\
\quad+K \sum_{n \in I_{N}} \gamma_{n}\left(x_{i}(t)-x_{n}(t)+x_{n}(t)-x_{j}(t)\right)^{T} \\
\quad \times\left(\nabla V_{0}\left(x_{n}(t)-x_{i}(t)\right)-\nabla V_{0}\left(x_{n}(t)-x_{j}(t)\right)\right) \\
\leq\left\|x_{i}(t)-x_{j}(t)\right\|\left\|b_{i}-b_{j}\right\|
\end{aligned}
$$

using the property (??). This results in

$$
\frac{\mathrm{d}\left(\left\|x_{i}(t)-x_{j}(t)\right\|\right)}{\mathrm{d} t} \leq\left\|b_{i}-b_{j}\right\|,
$$

and therefore

$$
\left\|\bar{v}_{i}-\bar{v}_{j}\right\|=\lim _{t \rightarrow+\infty} \frac{\left\|x_{i}(t)-x_{j}(t)\right\|}{t} \leq\left\|b_{i}-b_{j}\right\|
$$

This result implies that $\left\|\bar{v}_{m}-\bar{v}_{j}\right\|$, with $j \in H_{k}$, is upper bounded by $\min _{j \in H_{k}}\left\|b_{m}-b_{j}\right\|$. However, we may provide a stronger bound by considering a 'virtual agent': we may add an extra agent $N+1$ to the system with $\gamma_{N+1} \rightarrow 0$, since for $\gamma_{N+1}=0$ this agent will not influence the behavior of the other agents. Choosing $b_{N+1}$ in the convex hull of the natural 
velocities of the agents in $H_{k}$, it follows that $N+1 \in H_{k}$ and $\bar{v}_{N+1}=\bar{v}_{j}\left(j \in H_{k}\right)$, as shown in the previous section. As a consequence, if $j \in H_{k}$, then

$$
\left\|\bar{v}_{m}-\bar{v}_{j}\right\|=\left\|\bar{v}_{m}-\bar{v}_{N+1}\right\| \leq \min _{b_{N+1} \in \mathrm{CH}_{k}}\left\|b_{m}-b_{N+1}\right\|
$$

where $\mathrm{CH}_{k}$ denotes the convex hull of the natural velocities of the agents in $H_{k}$. In other words, $\left\|\bar{v}_{m}-\bar{v}_{j}\right\|$ is bounded by the distance of $b_{m}$ to the set $\mathrm{CH}_{k}$.

\section{SPHERICAL SYMMETRY WITH AN INFINITE NUMBER OF AGENTS}

We consider (??) with all-to-all coupling, with all weights $\gamma_{i}$ set equal to 1 , and with all the interaction functions $f_{i j}$ equal to some $f$, and thus also having a common limit value denoted by $F_{0}$. Furthermore we assume that the number of agents is infinite and consider a Lagrangian representation of the continuum limit of (??) with the natural velocities $b \in \mathbb{R}^{P}$ drawn from a distribution with a continuous probability density function $g: \mathbb{R}^{P} \rightarrow \mathbb{R}$. We propose that the dynamics are governed by the following partial differential equation:

$$
\frac{\partial x}{\partial t}(b, t)=b+\int_{\mathbb{R}^{P}} g\left(b^{\prime}\right) f\left(\left\|x\left(b^{\prime}, t\right)-x(b, t)\right\|\right) \mathrm{d} b^{\prime},
$$

for $x(b, t) \in \mathbb{R}^{P}, b \in \mathbb{R}^{P}$ and $t$ in $\mathbb{R}$. This setup has been introduced and explored before, for the case $P=1$ in $[?]$, to which the reader is referred for details.

We will present an in-depth study of the velocity clustering behavior of this system based on a continuous version of equation (??) established earlier for the finite case. (For convenience we may omit the 'velocity' qualification, when referring to clusters or clustering behavior.) We will assume that Lemma ?? can be extended to infinite $N$. As a consequence, agents with equal natural velocities $b$ belong to the same cluster; a cluster may then be represented by the region in $\mathbb{R}^{P}$ of the $b$-values of its agents. In this section the term 'cluster' has the same meaning as before, with the proviso that it is reserved for groups of agents for which this region for the $b$-values has a non-zero $P$-dimensional volume; this is a natural adaptation since we are working with an uncountable number of agents.

It turns out that a rigorous study of a continuous version of equation (??) is not trivial, even under the assumption of all-to-all coupling. We will therefore introduce a symmetry condition on the density $g$. Let $g(b)$ depend on $\|b\|$ only (i.e. $g(b)=g_{\mathrm{r}}(\|b\|)$, for all $b$ in $\mathbb{R}^{P}$, for some function $g_{\mathrm{r}}: \mathbb{R}^{+} \rightarrow \mathbb{R}$ ). Also without loss of generality, consider a spherically 
symmetric initial condition with $x(b, 0)=\alpha(\|b\|) b$ for some function $\alpha: \mathbb{R}^{+} \rightarrow \mathbb{R}$ (i.e. $x(b, 0)$ is parallel with $b$, with $\|x(b, 0)\|$ only depending on $\|b\|)$; the spherical symmetry will be maintained in the dynamics and therefore the velocities $\dot{x}(b, t)$ will only have a radial component (parallel with $b$ ). As a consequence the asymptotic velocities $\bar{v}(b)$ also have only a radial component, with magnitude denoted by $\bar{v}_{\mathrm{r}}(\|b\|)$. Since $\dot{x}_{i}(t)-\dot{x}_{j}(t)=b_{i}-b_{j}$ whenever $x_{i}(t)=x_{j}(t)$, it follows that $\bar{v}_{\mathrm{r}}$ is non-decreasing. Since Lemma ?? is assumed to be true for infinite $N, \bar{v}_{\mathrm{r}}$ is also continuous. By the spherical symmetry (and taking into account the restriction imposed on the use of the term 'cluster'), by the radial phase portrait of the asymptotic velocities (having different directions on different radii), and by the nondecreasing evolution of $\bar{v}_{\text {r }}$ it follows that for $P>1$ at most one cluster can be present: the cluster is associated with asymptotic velocity zero and contains all agents with $b$-values in a ball $B(0, \beta)$ with radius $\beta$ centered at the origin of $\mathbb{R}^{P}$. (If this cluster does not exist we set $\beta \triangleq 0$.) Interactions with agents outside this cluster are saturated (reaching their limit value $F_{0}$ ), as are the mutual interactions among agents (with different natural velocities) outside the cluster; from (??) it follows that the velocity function $\bar{v}$ satisfies

$$
\bar{v}(b)=b+K F_{0} \int_{\mathbb{R}^{P}} \mathrm{~d} b^{\prime} g\left(b^{\prime}\right) e_{\bar{v}\left(b^{\prime}\right)-\bar{v}(b)}, \quad \text { for all } b \text { in } \mathbb{R}^{P} \backslash B(0, \beta),
$$

and $\bar{v}(b)=0$ for all $b$ in $B(0, \beta)$.

\section{A. Cluster characterization}

Consider the limit $\|b\| \rightarrow \beta$ in (??). By continuity of $\bar{v}_{\mathrm{r}}, \bar{v}(b) \rightarrow 0$ ( since $\bar{v}_{\mathrm{r}}(\beta)=0$ ) and the interaction with agents outside the cluster will cancel because of the spherical symmetry:

$$
\lim _{\|b\| \rightarrow \beta} \int_{\mathbb{R}^{P} \backslash B(0, \beta)} \mathrm{d} b^{\prime} g\left(b^{\prime}\right) e_{\bar{v}\left(b^{\prime}\right)-\bar{v}(b)}=\int_{\mathbb{R}^{P} \backslash B(0, \beta)} \mathrm{d} b^{\prime} g\left(b^{\prime}\right) e_{\bar{v}\left(b^{\prime}\right)}=0 .
$$

Since we are approaching the edge of the cluster from the outside, the interaction with the agents inside the cluster is saturated and aligned with $0-\bar{v}(b)=-\bar{v}(b)$ :

$$
\lim _{\|b\| \rightarrow \beta} \int_{B(0, \beta)} \mathrm{d} b^{\prime} g\left(b^{\prime}\right) e_{\bar{v}\left(b^{\prime}\right)-\bar{v}(b)}=-\lim _{\|b\| \rightarrow \beta} e_{\widetilde{v}(b)} \int_{B(0, \beta)} \mathrm{d} b^{\prime} g\left(b^{\prime}\right) .
$$

It follows that

$$
0=\beta-K F_{0} \int_{B(0, \beta)} \mathrm{d} b^{\prime} g\left(b^{\prime}\right)
$$


This equation always has the solution $\beta=0$, which is relevant for the clustering behavior for sufficiently small values of $K$. For $K$ larger than some critical value $K_{\ell}$, there are also nonzero solutions for $\beta$. However, (??) only constitutes a necessary condition, and the existence of a non-zero solution for $\beta$ does not imply the existence of a cluster, as (??) only expresses the equilibrium between the natural velocity and the maximal centripetal attraction for an agent at the edge of the cluster, without considering agents inside the cluster. On the other hand, if there is a cluster, then it is (not necessarily uniquely) determined by the nonzero solution(s) for $\beta$ of (??). Therefore $K_{\ell}$ only establishes a lower bound for the critical coupling strength $K_{\mathrm{c}}$ at the onset of the cluster formation.

\section{B. Phase transition}

Equation (??) equates two terms related to opposite actions. While $\beta$ represents the magnitude of the natural velocity of an agent at the edge of the cluster - and therefore its tendency to move away from the cluster - , the term $K F_{0} \int_{B(0, \beta)} \mathrm{d} b^{\prime} g\left(b^{\prime}\right)$ corresponds to the maximal attraction that the entire cluster may exert on this agent.

For small values of $\beta$, Eq. (??) can be approximated by

$$
\beta \approx K F_{0} g(0) V_{P} \beta^{P}
$$

where $V_{P}$ denotes the volume of a $P$-dimensional ball with radius one. This approximation is reminiscent of classical descriptions of nucleation in thermodynamical phase transitions [? , p. 192]. E.g. the homogeneous nucleation of the condensed phase in supersaturated vapor is driven by a decrease in Gibb's free energy resulting from the substitution of vapor with liquid phase - proportional to the volume of the liquid phase - while being counteracted by an increase in free energy associated with the creation of an interface between vapor and liquid - proportional to the surface area of the liquid phase. For the free energy related to a ball of liquid with radius $R$, the two terms involved are proportional to $R^{3}$ and $R^{2}$ respectively, and their derivatives with respect to $R$ (proportional to $R^{2}$ and $R$ respectively) may be considered as two counteracting forces. At the value of $R$ where the Gibb's free energy is maximal, these forces are equal, leading to an unstable equilibrium. Above the corresponding critical radius the nucleus will grow further without requiring extra energy.

Although the dynamics of (??) and the mechanism inducing cluster formation may not 
be comparable to the nucleation process in thermodynamical systems, the behavior of both terms in (??) for small values of $\beta$ will affect the cluster formation in a similar way, with a transition similar to second-order or first-order phase transitions depending on whether $P=1$ or $P>1$.

\section{1. $P=1$}

The case $P=1$ has been investigated in [? ]. If $g_{\mathrm{r}}$ is decreasing in $\mathbb{R}^{+}$, then a typical graph of the cluster size $\int_{B(0, \beta)} \mathrm{d} b^{\prime} g\left(b^{\prime}\right)$ in terms of $\beta$ is shown in Fig. ??, and it follows that $K_{\mathrm{c}}=K_{\ell}=1 /\left(2 F_{0} g(0)\right)$. The cluster size grows continuously (but not differentiably) from 0 on with increasing $K$, according to (??) (see Fig. ??). If the cluster size is taken as an order parameter, its graph in terms of $K$ shows that the cluster appears according to a second-order phase transition.

If $g_{\mathrm{r}}$ is not decreasing everywhere in $\mathbb{R}^{+}$, then there are at least two local maxima of $g$, and two clusters will arise at $K=1 /\left(2 F_{0} \max (g)\right)$ as is discussed in [? ].

\section{2. $P>1$}

For $P>1$ a typical graph of the cluster size as a function of $\beta$ is shown in Fig. ??. There will be a minimum size for the cluster (as can also be inferred from (??)), resulting in a discontinuity for $\beta$ (and therefore also for the cluster size) in terms of $K$, similar to the discontinuities observed in first-order phase transitions (see Fig. ??). (In contrast with the nucleation process in thermodynamical systems, the cluster growth will be limited for fixed $K$ because the second term in Eq. (??) contains higher order terms not present in the approximation (??), and the cluster size will be equal to the largest solution of (??).)

Remark 5. A similar discontinuity of the cluster size as a function of $K$ may also occur for $P=1$ if the density function is constant in an interval with non-zero length, but this is a non-generic situation (see also [?]). 


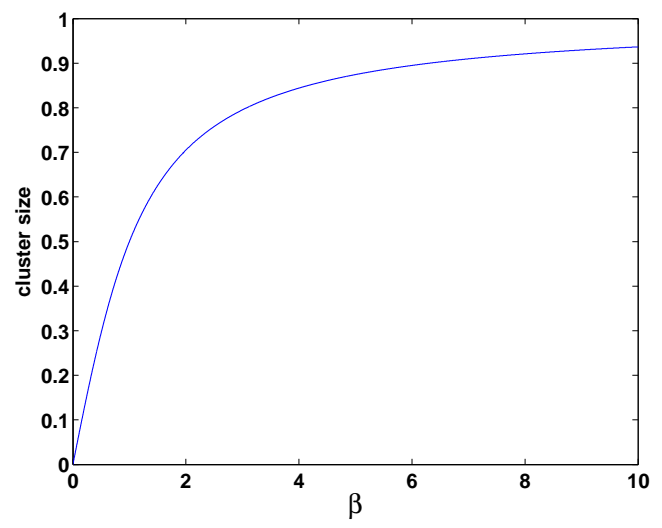

(a) $P=1$

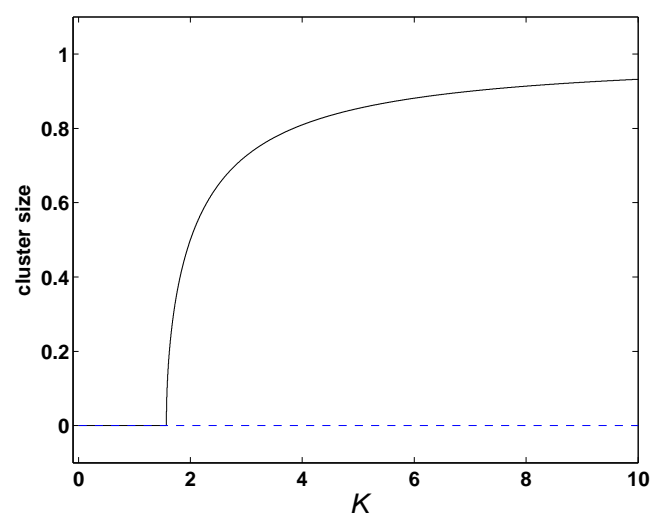

(c) $P=1$

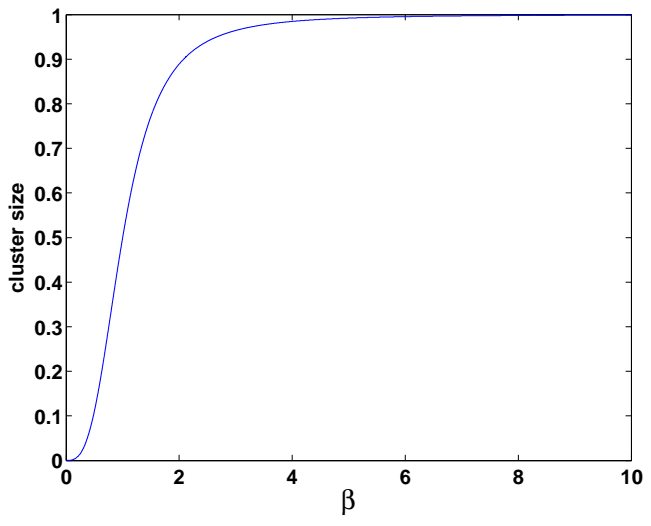

(b) $P=3$

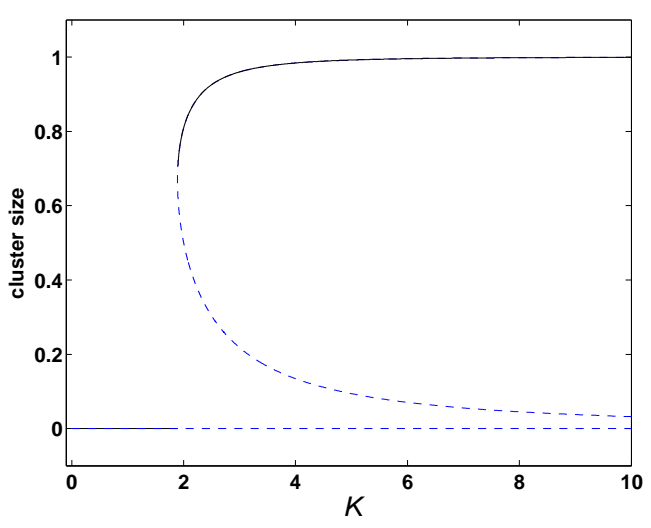

(d) $P=3$

Figure 2. Comparison of the one-dimensional case with the multi-dimensional case for the evolution of the cluster size for typical choices of the (unimodal) density function $g$. The upper row shows the cluster size in terms of $\beta$; the lower row shows the evolution of the cluster size in terms of the coupling strength $K$ when solving (??) (the part of the graph corresponding to the clusters in the dynamical system is shown with a solid line). The left column corresponds to $P=1$, the right column to $P=3$. (For $P=3$, the density function is taken equal to $g_{1}$ in section ??.)

\section{Bounds for the critical coupling strength}

The value $K_{\ell}$ (i.e. the infimum of all $K$-values for which (??) has a non-zero solution for $\beta$ ) constitutes a lower bound for $K_{\mathrm{c}}$. It satisfies

$$
1=K_{\ell} F_{0} \max _{r \in \mathbb{R}_{0}^{+}} \frac{\int_{B(0, r)} \mathrm{d} b g(b)}{r} .
$$


An upper bound for $K_{\mathrm{c}}$ can be derived as follows.

In the integral in (??) we can first perform an integration over hyperspheres with radius $r^{\prime}$, and then integrate over $r^{\prime}$. With $A_{P}$ denoting the $P$-1-dimensional surface area of a unit hypersphere in $\mathbb{R}^{P}$, this leads to

$$
\bar{v}_{\mathrm{r}}(r)=r-K F_{0} \int_{0}^{+\infty} \mathrm{d} r^{\prime} A_{P} r^{\prime P-1} g_{\mathrm{r}}\left(r^{\prime}\right) \Phi_{P}\left(\frac{\bar{v}_{\mathrm{r}}(r)}{\bar{v}_{\mathrm{r}}\left(r^{\prime}\right)}\right), \quad \text { for all } r \text { in }[\beta,+\infty),
$$

where $\Phi_{P}\left(\frac{\bar{v}_{\mathrm{r}}(r)}{\bar{v}_{\mathrm{r}}\left(r^{\prime}\right)}\right)$ represents the attraction of agents on a sphere (centered at the origin) with radius $\bar{v}_{\mathrm{r}}\left(r^{\prime}\right) t$ (with $t$ tending to $+\infty$ ) on an agent $\bar{v}_{\mathrm{r}}(r) t$ away from the origin. It can be written as

$$
\Phi_{P}(\xi) \triangleq \frac{A_{P-1}}{A_{P}} \int_{0}^{\pi} \mathrm{d} \alpha(\sin \alpha)^{P-2} \frac{\xi-\cos \alpha}{\sqrt{\xi^{2}-2 \xi \cos \alpha+1}}, \quad \text { for all } \xi \text { in } \mathbb{R}^{+},
$$

where $A_{P}$ satisfies

$$
A_{P}=A_{P-1} \int_{0}^{\pi} \mathrm{d} \alpha(\sin \alpha)^{P-2},
$$

with $A_{1}=2$. The function $\Phi_{P}$ is increasing (the derivative of the integrand to $\xi$ is positive for almost all $\alpha$ in $[0, \pi])$ and satisfies $\Phi_{P}(0)=0$ and $\lim _{\xi \rightarrow+\infty} \Phi_{P}(\xi)=1$. Since $\bar{v}_{\text {r }}$ is non-decreasing, it follows that $\Phi_{P}\left(\frac{\bar{v}_{\mathrm{r}}(r)}{\bar{v}_{\mathrm{r}}\left(r^{\prime}\right)}\right)$ is non-increasing in $r^{\prime}$, and using (??) we derive that

$$
\begin{aligned}
\bar{v}_{\mathrm{r}}(r) & \leq r-K F_{0} \int_{0}^{r} \mathrm{~d} r^{\prime} A_{P} r^{\prime P-1} g_{\mathrm{r}}\left(r^{\prime}\right) \Phi_{P}(1), \\
& =r-\Phi_{P}(1) K F_{0} \int_{B(0, r)} \mathrm{d} b g(b),
\end{aligned}
$$

for all $r$ in $[\beta,+\infty)$, and thus, since if $K=K_{\mathrm{c}}$ then $\beta=0$,

$$
r-\Phi_{P}(1) K_{\mathrm{c}} F_{0} \int_{B(0, r)} \mathrm{d} b g(b) \geq 0, \quad \text { for all } r \text { in } \mathbb{R}^{+} .
$$

Together with the previously derived lower bound for $K_{\mathrm{c}}$, we can conclude that

$$
1 \leq K_{\mathrm{c}} F_{0} \max _{r \in \mathbb{R}_{0}^{+}} \frac{\int_{B(0, r)} \mathrm{d} b g(b)}{r} \leq \frac{1}{\Phi_{P}(1)},
$$

with

$$
\Phi_{P}(1)=\frac{\int_{0}^{\pi} \mathrm{d} \alpha(\sin \alpha)^{P-2} \sin \frac{\alpha}{2}}{\int_{0}^{\pi} \mathrm{d} \alpha(\sin \alpha)^{P-2}} .
$$

The bounds on $K_{\mathrm{c}}$ remain non-trivial for $P \rightarrow+\infty$, since $\lim _{P \rightarrow+\infty} \Phi_{P}(1)=\frac{1}{\sqrt{2}}$. This can be verified by noticing that for large values of $P$, the main contribution to the integrals in the expression for $\Phi_{P}(1)$ stems from integration over a small interval about $\frac{\pi}{2}$. 
Remark 6 . The upper bound is reached for a density function $g$ of the form

$$
g(b)=\frac{1}{A_{P} C^{P-1}} \delta(\|b\|-C),
$$

for some $C>0$, where $\delta$ represents the (one-dimensional) Dirac distribution. This distribution is obviously not continuous. As we will illustrate below for $P=3$, there may exist continuous distributions for which the lower bound is attained.

D. Example: $P=3$

The function $\Phi_{3}$ may be written in explicit form as follows:

$$
\begin{aligned}
\Phi_{3}(\xi) & =\frac{1}{2} \int_{-1}^{1} \mathrm{~d} u \frac{\xi-u}{\sqrt{\xi^{2}-2 \xi u+1}} \quad(u=\cos \alpha) \\
& =\frac{1}{2} \int_{|\xi-1|}^{\xi+1} \mathrm{~d} z \frac{\xi^{2}-1+z^{2}}{2 \xi^{2}} \quad\left(z=\sqrt{\xi^{2}-2 \xi u+1}\right) \\
& =\frac{1}{4 \xi^{2}}\left(\left(\xi^{2}-1\right)(\xi+1-|\xi-1|)+\frac{1}{3}\left((\xi+1)^{3}-|\xi-1|^{3}\right)\right) \\
& = \begin{cases}\frac{2}{3} \xi, & \text { for } \xi \text { in }[0,1] ; \\
1-\frac{1}{3 \xi^{2}}, & \text { for } \xi \text { in }[1,+\infty) .\end{cases}
\end{aligned}
$$

Substituting this expression in (??) results in

$$
\begin{aligned}
\bar{v}_{\mathrm{r}}(r)=r & -K F_{0} \int_{0}^{r} \mathrm{~d} r^{\prime} 4 \pi r^{\prime 2} g_{\mathrm{r}}\left(r^{\prime}\right)\left(1-\frac{\bar{v}_{\mathrm{r}}\left(r^{\prime}\right)^{2}}{3 \bar{v}_{\mathrm{r}}(r)^{2}}\right) \\
& -K F_{0} \int_{r}^{+\infty} \mathrm{d} r^{\prime} \frac{8 \pi r^{\prime 2}}{3} g_{\mathrm{r}}\left(r^{\prime}\right) \frac{\bar{v}_{\mathrm{r}}(r)}{\bar{v}_{\mathrm{r}}\left(r^{\prime}\right)},
\end{aligned}
$$

for all $r$ in $[\beta,+\infty)$. Dividing by $\bar{v}_{\mathrm{r}}(r)$ and taking the derivative with respect to $r$ leads to

$$
\begin{aligned}
0=\frac{1}{\bar{v}_{\mathrm{r}}(r)}- & \frac{r \frac{\mathrm{d} \bar{v}_{\mathrm{r}}}{\mathrm{d} r}(r)}{\bar{v}_{\mathrm{r}}(r)^{2}}+\frac{\frac{\mathrm{d} \bar{v}_{\mathrm{r}}}{\mathrm{d} r}(r)}{\bar{v}_{\mathrm{r}}(r)^{2}} K F_{0} \int_{0}^{r} \mathrm{~d} r^{\prime} 4 \pi r^{\prime 2} g_{\mathrm{r}}\left(r^{\prime}\right)-\frac{K F_{0}}{\bar{v}_{\mathrm{r}}(r)} 4 \pi r^{2} g_{\mathrm{r}}(r) \\
& \quad-3 \frac{\frac{\mathrm{d} \bar{v}_{\mathrm{r}}}{\mathrm{d} r}(r)}{\bar{v}_{\mathrm{r}}(r)^{4}} K F_{0} \int_{0}^{r} \mathrm{~d} r^{\prime} \frac{4}{3} \pi r^{\prime 2} g_{\mathrm{r}}\left(r^{\prime}\right) \bar{v}_{\mathrm{r}}\left(r^{\prime}\right)^{2}+\frac{K F_{0}}{\bar{v}_{\mathrm{r}}(r)} \frac{4}{3} \pi r^{2} g_{\mathrm{r}}(r)+\frac{K F_{0}}{\bar{v}_{\mathrm{r}}(r)} \frac{8 \pi r^{2}}{3} g_{\mathrm{r}}(r),
\end{aligned}
$$

or

$$
\begin{aligned}
0=\frac{1}{\bar{v}_{\mathrm{r}}(r)}-\frac{r \frac{\mathrm{d} \overline{\mathrm{v}}_{\mathrm{r}}}{\mathrm{d} r}(r)}{\bar{v}_{\mathrm{r}}(r)^{2}}+\frac{\frac{\mathrm{d} \bar{v}_{\mathrm{r}}}{\mathrm{d} r}(r)}{\bar{v}_{\mathrm{r}}(r)^{2}} K F_{0} \int_{0}^{r} \mathrm{~d} r^{\prime} 4 \pi r^{\prime 2} g_{\mathrm{r}}\left(r^{\prime}\right) & \\
& -\frac{\frac{\mathrm{d} \overline{\mathrm{v}}_{\mathrm{r}}(r)}{\mathrm{d} r} \bar{v}_{\mathrm{r}}(r)^{4}}{} K F_{0} \int_{0}^{r} \mathrm{~d} r^{\prime} 4 \pi r^{\prime 2} g_{\mathrm{r}}\left(r^{\prime}\right) \bar{v}_{\mathrm{r}}\left(r^{\prime}\right)^{2}
\end{aligned}
$$


Multiplying with $\frac{\bar{v}_{\mathrm{r}}(r)^{4}}{\frac{\mathrm{d} \overline{\mathrm{d}}_{\mathrm{r}}}{\mathrm{d} r}(r)}$ and again taking the derivative to $r$, we obtain

$$
\begin{aligned}
0=3 & \bar{v}_{\mathrm{r}}(r)^{2}-\frac{\bar{v}_{\mathrm{r}}(r)^{3} \frac{\mathrm{d}^{2} \bar{v}_{\mathrm{r}}}{\mathrm{d} r^{2}}(r)}{\left(\frac{\mathrm{d} \bar{v}_{\mathrm{r}}}{\mathrm{d} r}(r)\right)^{2}}-\bar{v}_{\mathrm{r}}(r)^{2}-2 r \bar{v}_{\mathrm{r}}(r) \frac{\mathrm{d} \bar{v}_{\mathrm{r}}}{\mathrm{d} r}(r) \\
& +2 \bar{v}_{\mathrm{r}}(r) \frac{\mathrm{d} \bar{v}_{\mathrm{r}}}{\mathrm{d} r}(r) K F_{0} \int_{0}^{r} \mathrm{~d} r^{\prime} 4 \pi r^{\prime 2} g_{\mathrm{r}}\left(r^{\prime}\right)+\bar{v}_{\mathrm{r}}(r)^{2} K F_{0} 4 \pi r^{2} g_{\mathrm{r}}(r)-K F_{0} 4 \pi r^{2} g_{\mathrm{r}}(r) \bar{v}_{\mathrm{r}}(r)^{2},
\end{aligned}
$$

or

$$
\frac{\bar{v}_{\mathrm{r}}(r)}{\frac{\mathrm{d} \bar{v}_{\mathrm{r}}}{\mathrm{d} r}(r)}-\frac{\bar{v}_{\mathrm{r}}(r)^{2} \frac{\mathrm{d}^{2} \bar{v}_{\mathrm{r}}}{\mathrm{d} r^{2}}(r)}{2\left(\frac{\mathrm{d} \bar{v}_{\mathrm{r}}}{\mathrm{d} r}(r)\right)^{3}}=U(r),
$$

where

$$
U(r) \triangleq r-K F_{0} \int_{0}^{r} \mathrm{~d} r^{\prime} 4 \pi r^{\prime 2} g_{\mathrm{r}}\left(r^{\prime}\right)
$$

for all $r$ in $\mathbb{R}^{+}$. Setting $\chi \triangleq \frac{\bar{v}_{r}}{\frac{d \bar{v}_{r}}{d r}}$, it follows that

$$
\frac{\mathrm{d} \chi}{\mathrm{d} r}=\frac{2 U-\chi}{\chi}
$$

From the definition of $\chi$ we derive that

$$
\bar{v}_{\mathrm{r}}(r)=\bar{v}_{\mathrm{r}}\left(r_{0}\right) \exp \left(\int_{r_{0}}^{r} \frac{\mathrm{d} r^{\prime}}{\chi\left(r^{\prime}\right)}\right),
$$

with $r_{0} \in[\beta,+\infty)$. Since $\bar{v}_{\mathrm{r}}$ is non-negative and non-decreasing, with $\bar{v}_{\mathrm{r}}(\beta)=0$, it follows that $\chi$ is also non-negative, with $\lim _{r \rightarrow \beta} \chi(r)=0$. To investigate the existence of a cluster we can therefore consider a solution $\mathcal{S}$ of the system

$$
\begin{aligned}
\dot{r}(t) & =\chi(t), \\
\dot{\chi}(t) & =2 U(r(t))-\chi(t),
\end{aligned}
$$

with an initial condition $\left(r_{1}, \chi_{1}\right) \in\left(\mathbb{R}_{0}^{+}\right)^{2}$ near $(0,0)$. (Since the origin is a saddle point with the unstable manifold along the direction $(1,1)$, the exact value of the initial condition is unimportant, as long as $\left(r_{1}, \chi_{1}\right)$ is small enough.) If the solution for $\chi$ remains positive (notice that $r$ then also remains positive), then the corresponding $\bar{v}_{\mathrm{r}}$ solves (??) with $\beta=0$, and there will not be any cluster. If $\chi(t)=0$ for some $t>0$, then there is a cluster of which the size is determined by Eq. (??), or equivalently $U(\beta)=0$. To calculate $\bar{v}_{\mathrm{r}}$ in $[\beta,+\infty)$ one may consider an appropriate solution of (??) starting near $(\beta, 0)$.

As a first illustration we consider the density function $g_{1}$ defined by

$$
g_{1}(b)=\frac{3}{4 \pi\left(1+\|b\|^{3}\right)^{2}}, \quad \text { for all } b \text { in } \mathbb{R}^{P},
$$


such that

$$
\int_{0}^{r} \mathrm{~d} r^{\prime} 4 \pi r^{\prime 2} g_{\mathrm{r}}\left(r^{\prime}\right)=\frac{r^{3}}{1+r^{3}}, \quad \text { for all } r \text { in } \mathbb{R}^{+}
$$

Since

$$
\sup _{r \in \mathbb{R}_{0}^{+}} \frac{\int_{0}^{r} \mathrm{~d} r^{\prime} 4 \pi r^{\prime 2} g_{\mathrm{r}}\left(r^{\prime}\right)}{r}=\frac{4^{1 / 3}}{3},
$$

and $\Phi_{3}(1)=\frac{2}{3}$, the inequalities (??) result in

$$
\frac{3}{2^{2 / 3}} \leq K_{\mathrm{c}} F_{0} \leq \frac{9}{2^{5 / 3}}
$$

or, numerically approximated,

$$
1.8899 \leq K_{\mathrm{c}} F_{0} \leq 2.8348
$$

A simulation of $\mathcal{S}$ corresponding to $K F_{0}=1.897$ is shown in Fig. ??, together with the graph of the relation $\chi=U(r)$. As the trajectory almost touches the $r$-axis at $r \approx 1.34$ (which constitutes the largest solution of $U(r)=0$ ), we can conclude that this value of $K$ is still below, but very near to, $K_{\mathrm{c}}$. Notice that there is no cluster in this case, even though Eq. (??) has non-zero solutions for $\beta$.

In a second illustration we consider the density function $g_{2}$ defined by

$$
\int_{B(0, r)} g_{2}(b) \mathrm{d} b=\min \left\{r^{3}, r / C, 1\right\}, \quad \text { for all } r \text { in } \mathbb{R}^{+},
$$

for some $C>1$. We will show that for $C$ sufficiently large the lower bound in (??) is attained. (The corresponding density function $g_{2}$ is not continuous, but we assume that the results formulated below are still valid. Furthermore, one may approximate $g_{2}$ by a continuous distribution for which the lower bound in (??) is also attained, but the corresponding expression for the density function may be more complex.) The lower bound in (??) leads to

$$
K_{\mathrm{c}} F_{0} \geq C
$$

For $K F_{0}=C$ the function $U$ satisfies

$$
U(r)=0, \quad \text { for all } r \text { in }\left[\frac{1}{\sqrt{C}}, C\right]
$$

and a solution of the system (??) with $r(t) \in\left[\frac{1}{\sqrt{C}}, C\right]$ then satisfies

$$
\frac{\dot{\chi}(t)}{\dot{r}(t)}=-1 \text {. }
$$




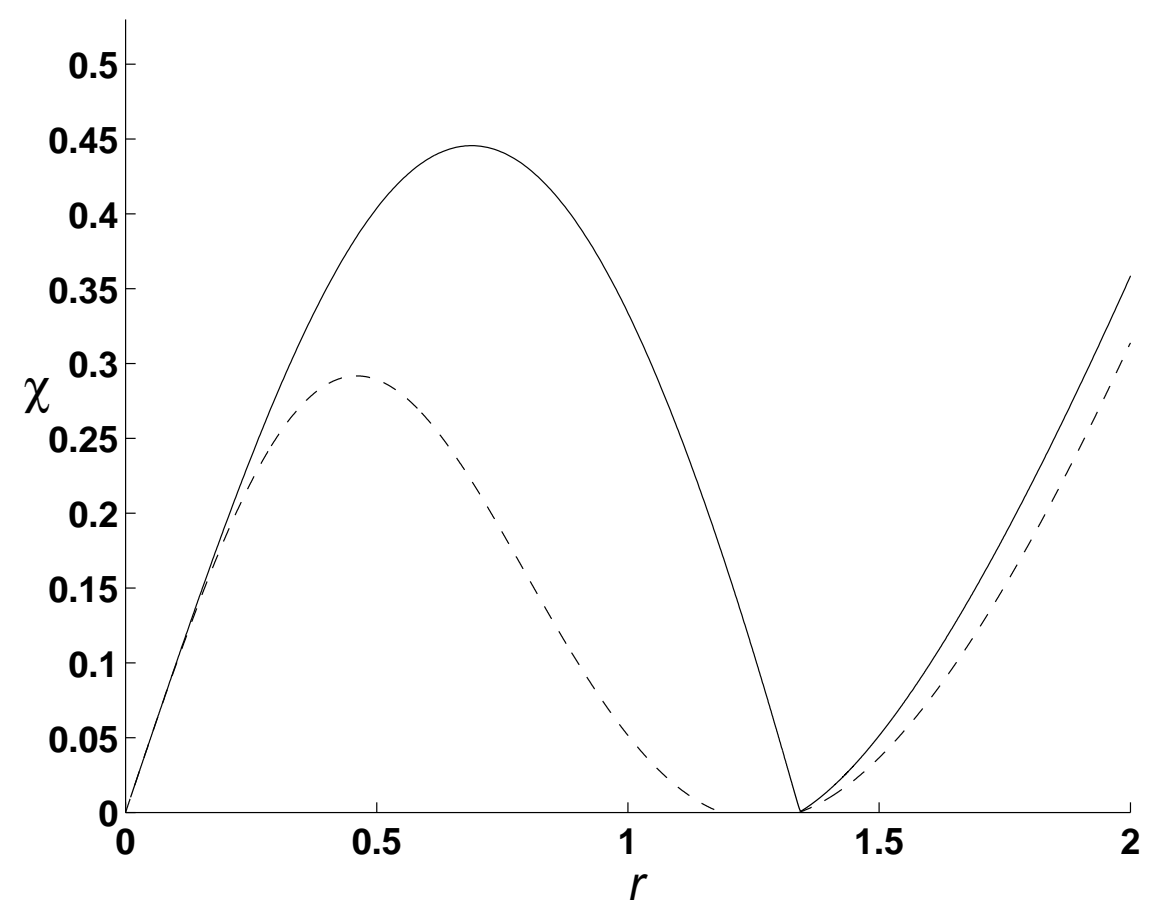

Figure 3. The trajectory of $\mathcal{S}$ is shown (solid line), together with the graph of the function $U$ (dashed line) for the density function $g_{1}$, and with $K F_{0}=1.897$.

Since

$$
\frac{\mathrm{d} \chi}{\mathrm{d} r} \leq \frac{2 r-\chi}{\chi}
$$

the first part of $\mathcal{S}$ (more specifically, the part for which $r(t) \leq \frac{1}{\sqrt{C}}$ ) is bounded by the solution of

$$
\frac{\mathrm{d} \chi}{\mathrm{d} r}=\frac{2 r-\chi}{\chi}
$$

starting near the origin, which corresponds to $\chi=r$, it follows that for $C$ sufficiently large and $K F_{0}=C, \mathcal{S}$ will cross the $r$-axis somewhere between $\frac{1}{\sqrt{C}}$ and $C$, and thus there is a cluster for this value of $K$, implying that $K_{\mathrm{c}} F_{0} \leq C$, and therefore $K_{\mathrm{c}} F_{0}=C$. Figure ?? shows a similar picture as Fig. ??, but for the density function $g_{2}$ and with $K F_{0}=C=$ 1.2985. Although this value of $K$ is still smaller than $K_{\mathrm{c}}$ (as can be concluded from Fig. ??) and thus in this case $K_{\mathrm{c}} F_{0}>C, K_{\mathrm{c}} F_{0}$ is very near to $C$, and simulations indicate that for slightly larger values of $C$ the equality $K_{\mathrm{c}} F_{0}=C$ holds. 


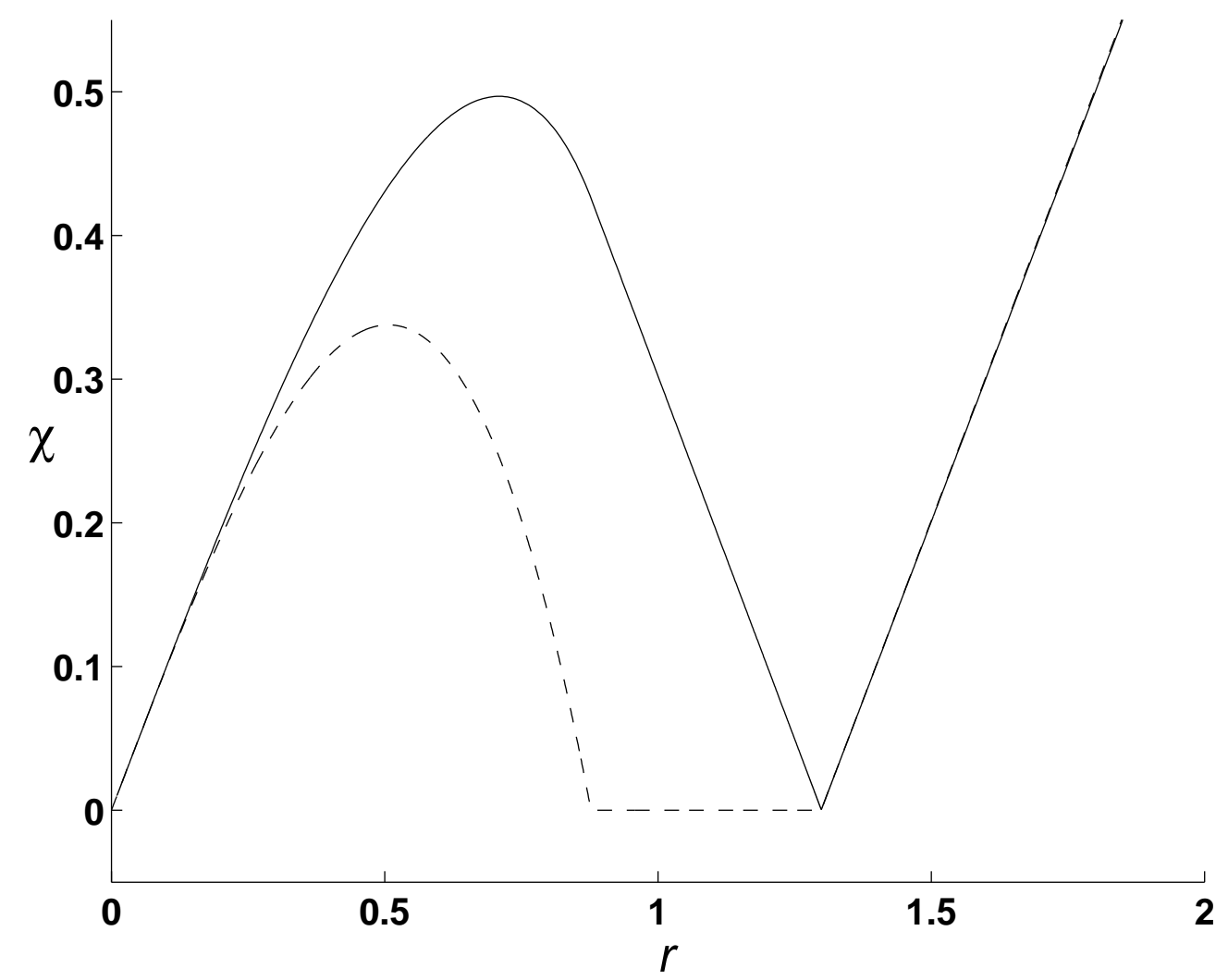

Figure 4. The trajectory of $\mathcal{S}$ is shown (solid line), together with the graph of the function $U$ (dashed line) for the density function $g_{2}$, and with $K F_{0}=C=1.2985$.

\section{CONCLUSION}

We have considered a multi-dimensional multi-agent clustering model for which the behavior of the agents is determined by non-identical natural velocities and interactions that may be represented as gradients of convex functions. We have shown that the velocities of the agents converge to limit values, constituting the solution to a minimization problem. The agents organize themselves into clusters, which may be defined by either bounded distances between the agents, or by a common asymptotic velocity. The definitions coincide for generic values of the parameters, and for both definitions the clustering behavior is independent of the initial conditions. If the interaction is all-to-all and identical for each pair of agents, then it is shown that the clusters are convex with respect to the natural velocities.

We have investigated the continuum limit of the model based on continuous versions of results obtained and presented in Section ?? for the finite number of agents case. As- 
suming spherical symmetry, and we have compared the emergence of a cluster for the onedimensional case with the higher-dimensional case. For the one-dimensional case the onset of cluster formation is similar to a second-order phase transition; in higher dimensions there is a minimal size for the cluster, and its emergence is akin to a first-order phase transition. Furthermore, we have provided bounds for the critical coupling strength at the onset of the cluster formation, and we have illustrated our results with two examples in three dimensions.

\section{ACKNOWLEDGEMENTS}

This paper presents research results of the Belgian Network DYSCO (Dynamical Systems, Control, and Optimization), funded by the Interuniversity Attraction Poles Programme, initiated by the Belgian State, Science Policy Office. The scientific responsibility rests with its authors.

This research was supported by the Research Foundation - Flanders (FWO) through the project G.0026.06N. 